This item was submitted to Loughborough's Research Repository by the author.

Items in Figshare are protected by copyright, with all rights reserved, unless otherwise indicated.

\title{
Twisting techniques used by competitive divers
}

PLEASE CITE THE PUBLISHED VERSION

http://dx.doi.org/10.1080/02640419308730003

PUBLISHER

Taylor \& Francis $@$ British Association of Sport and Exercise Sciences

VERSION

NA (Not Applicable or Unknown)

LICENCE

CC BY-NC-ND 4.0

REPOSITORY RECORD

Yeadon, Maurice R.. 2019. "Twisting Techniques Used by Competitive Divers". figshare. https://hdl.handle.net/2134/11675. 
This item was submitted to Loughborough's Institutional Repository (https://dspace.lboro.ac.uk/) by the author and is made available under the following Creative Commons Licence conditions.

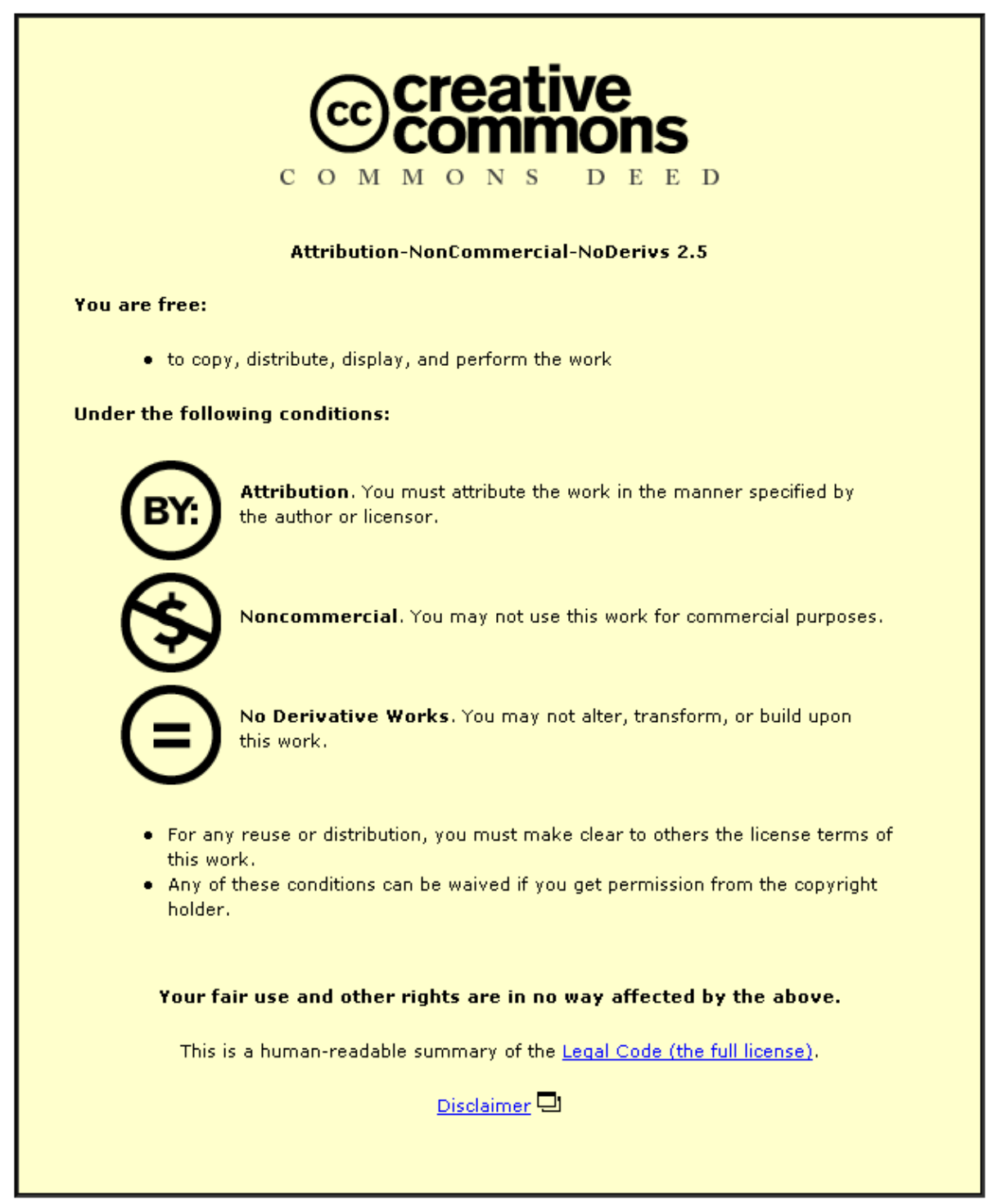

For the full text of this licence, please go to: http://creativecommons.org/licenses/by-nc-nd/2.5/ 


\title{
Twisting techniques used by competitive divers
}

\author{
M.R. Yeadon \\ Department of Physical Education, Sports Science and Recreation Management, \\ Loughborough University of Technology, Loughborough, LE11 3TU, UK
}

\begin{abstract}
At the 1991 World Student Games, eight reverse $1 \frac{1}{2}$ somersault dives with $2 \frac{1}{2}$ twists were recorded during the men's finals in the $1 \mathrm{~m}$ and $3 \mathrm{~m}$ springboard diving competitions using two video cameras. Angles describing body configuration were determined from video data and were input, together with initial orientation angle values and angular momentum components, into a computer simulation model of aerial movement in order to predict body orientation in space. Mean absolute deviations between simulation and video after the completion of one twist were $0.02 \mathrm{rev}$ for somersault, $2.3^{\circ}$ for tilt and 0.04 rev for twist. Contributions to the tilt angle after one twist were used as measures of the twisting potential of various techniques and were determined using simulations based on modifications of the video data. Seven of the eight competitors produced the majority of the tilt using aerial techniques which were predominantly asymmetrical movements of the arms and hips, although the mean contribution from contact techniques amounted to one-third of the total tilt.
\end{abstract}

Keywords: Diving, twisting, somersault, simulation, model.

\section{INTRODUCTION}

Twisting somersaults comprise the most technically difficult group of springboard dives. In non-twisting dives, the diver must leave the board with the appropriate linear and somersaulting veloctities and must then adjust body configuration during flight to obtain the correct somersault angle at entry into the water. In twisting dives, in addition to meeting the above requirements, the diver must also produce the required amount of twist and adjust both somersault and twist prior to entry.

Twisting techniques may be divided into those that start the twist during the contact with the board and those that initiate the twist during the aerial phase. This gives rise to the terms 'contact techniques' and 'aerial techniques' for the production of twist. McCormick(1954), Winter (1966) and Sanders and Wilson (1986) conducted descriptive studies of twisting dives and concluded that aerial techniques were responsible for the production of twist, whereas Mood (1968) found that the twisting rotation of the trunk started during the contact phase. Such cinematographical studies of twisting somersault can establish the orientations of the body throughout a movement together with the relative configurations of body segments. This information may suggest that a particular type of twist (e.g. contact, aerial) was used, but without a quantitative mechanical analysis the conclusions that can be drawn are limited. If a diver has completed some of the twist and has a twisting velocity when the feet leave the board, it may seem reasonable to conclude that the twisting technique employed by the diver was one of starting the twist during the contact phase. Such a conclusion would be premature. If a somersault without twist is analysed, the amounts of twist and twisting velocity at take-off will not be eactly zero and so it is a matter of the mechanical significance of these values. Even if the diver does have a substantial contribution from the contact phase, this contribution may not be the major one. Additionally, any actual performance will exhibit configurational changes which may be interpreted as showing that a particular technique contributes to the twist. Without some way of quantifying the contributions of each technique in a twisting somersault, any conclusion reached on the origin of the twist is likely to reflect the investigator's personal opinions. As McDonald (1961) wrote: '... how easy it is to have a preconceived theory and interpret what you see so that it fits'. Thus, although the above investigations attempted to 
determine the twisting techniques used, none used a mechanical evaluation and the results presented are open to other interpretations.

In the study of Lanoue (1936), several experienced divers dropped in various positions from a height of $5 \mathrm{~m}$ and attempted to twist after release. They had little success unless they were aided by an initial impulse during the contact phase. In contrast, McDonald (1961) showed that an elite diver could produce a full twist in jumps from the $1 \mathrm{~m}$ springboard. Bartee and Dowell (1982) used a swivel board to ensure that no twist was produced during the take-off from the diving platform. They concluded that it was indeed possible to produce twist during the aerial phase of a plain somersault but were unable to establish the techniques responsible. Although these experimental studies show that it is possible to produce twist after leaving the diving board, the actual techniques used have not been identified.

Pike (1980) used a five-segment computer simulation model to show that a full twist could be introduced into a plain dive by means of asymmetrical arm movements during the aerial phase. Some caution should be exercised when viewing the results obtained from such a model whose accuracy is unknown. Of the various models used in theoretical studies of twisting somersaults, only those of Van Gheluwe (1981) and Yeadon et al. (1990) have been shown to be accurate representations of actual performances. Van Gheluwe (1981) was able to show that, in three performances of full twisting backward somersaults on a trampoline, the twist resulted mainly from the arm movements in the aerial phase. Yeadon and Dunn (1987) used an 11-segment simulation model to analyse eight backward and reverse somersaults with $1 \frac{1}{2}$ and $2 \frac{1}{2}$ twists from the $1 \mathrm{~m}$ springboard and found that the majority of the twist arose from asymmetrical movements made during the aerial phase.

No study has yet addressed the question of which techniques are used by elite divers in competition. This paper will investigate the techniques used in competitive performances of reverse $1 \frac{1}{2}$ somersaults with $2 \frac{1}{2}$ twists.

\section{Method}

\section{Data collection}

Video-recordings were made of the men's $1 \mathrm{~m}$ and $3 \mathrm{~m}$ diving competitions at the World Student Games in Sheffield. Two Panasonic MS2 SVHS cameras recorded the twisting dives performed in these two competitions from 18 to 22 July 1991. The camera locations were chosen so that the camera axes were approximately perpendicular (as shown in Figure 1) for the $3 \mathrm{~m}$ springboard competition. The field of view of each camera was $7 \mathrm{~m} \times 5 \mathrm{~m}$ and covered that part of the flight phase above the level of the springboard. Since the cameras recorded 50 fields per second, this resulted in between 50 and 60 fields for each dive. The cameras were used with electronic $1 / 250$ s shutters in order to give a sharp image of rapidly moving body segments. Distances were taken using a measuring tape and were used in conjunction with scale plans of the facility to establish the three-dimensional locations of the cameras and diving boards for later use in the camera calibration. The heights of the cameras above the diving pool were estimated by visually siting along the surface of the $10 \mathrm{~m}$ platform and the measuring down from this level.

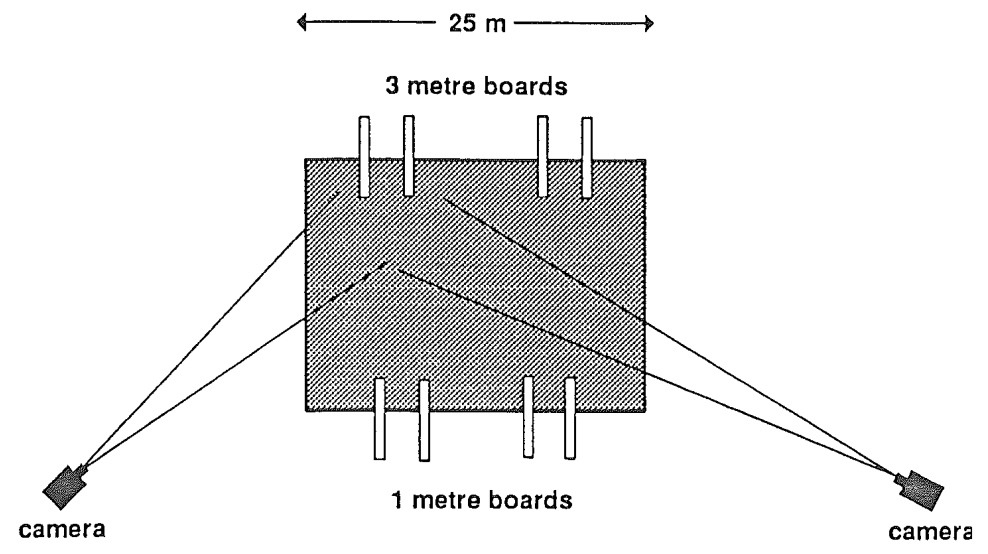

Figure 1: Location of cameras in relation to the diving pool. 


\section{Data analysis}

The divers who used the reverse $1 \frac{1}{2}$ somersault with $2 \frac{1}{2}$ twists in the finals of the competitions were identified. From these the three finalists of the $3 \mathrm{~m}$ competition and the five highest scoring competitors in the $1 \mathrm{~m}$ finals were selected for analysis. This resulted in competitors from Australia, Canada, China, Japan and the USA.

The two views of the aerial phase of each dive were projected onto a TDS HR48 Tablet Digitizer using an Imager LCD10E video projector. The corners of the projected video image were each digitized four times and their mean co-ordinate values were taken to give the centre of the projected video field. The images of two points on the front edges of the two springboards in view were each digitized eight times and mean co-ordinate values obtained in a field prior to the diver advancing down the board. From the measured three-dimensional locations of the two cameras and the two reference points on the diving boards together with the digitized data, the camera calibration was carried out using the method of Yeadon (1989a).

In each video field of the aerial phase, the wrist, elbow, shoulder, hip, knee, and ankle centres were digitized, giving 12 landmarks in total. The head was assumed to be in normal alignment and the ankles were assumed to be plantar-flexed. Each view of a dive was digitized twice to facilitate error correction and to produce error estimates for use in curve-fitting the coordinate data using the quintic spline of Wood and Jennings (1979). These mathematical fits to the digitized data permitted the estimation of data valuees at times in between those of the video fields. The digitized data sets from the two cameras were synchronized by determining rays from each camera to an unweighted mean position of the 12 digitized points for the first three and last three fields from each camera. The method described in Yeadon (1989a) was used to determine the time of the second and penultimate field from one camera in terms of the time-scale of the other camera. The quintic spline fits to the displacement data were then used to obtain interpolated digitized values to produce synchronous data sets.

The body was modelled using four arm segments, four leg segments and three torso segments. The orientation of the body was defined by angles corresponding to somersault, tilt and twist, where somersault is the rotation about an axis parallel to the angular momentum vector, twist is the rotation about the longitudinal body axis corresponding to minimum moment of inertia, and tilt is the angle between the longitudinal axis and the plane perpendicular to the angular momentum vector (Fig 2). Body configuration was specified using 14 angles to descibe the relative orientations of the body segments arising from movement at the elbows, shoulders, hips, knees and two points on the spine (Yeadon 1990c). The three-dimensional co-ordinates of the digitized data (Yeadon, 1989a) and the time histories of the orientation and configuration angles were determined (Yeadon, 1990a).

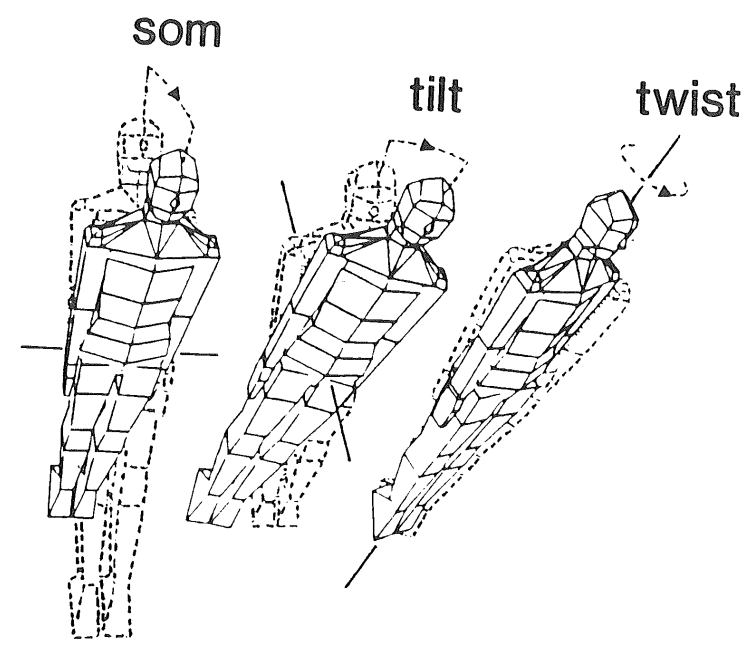

Figure 2: Angles of somersault, tilt and twist.

A series of 95 antrhopometric measurements (lengths, widths, depths and perimeters) defined in Yeadon (1990b) were taken on four divers in a previous study (Yeadon, 1989b). Two of these divers 
(numbers 523 and 529) were among the eight selected for analysis in this study. Segmental masses, mass centre locations, link lengths and moments of inertia were determined from these four data sets using the mathematical inertia model of Yeadon (1990b).

An 11-segment model was used to determine the angular momentum vector during each dive for each of the four sets of segmental inertia parameters using the time histories of the 17 orientation and configuration angles (Yeadon, 1990c). The mean values of the calculated angular momentum components and the time histories of the 14 configurational angles, together with the initial values of the three orientation angles and the segmental inertia parameters, were used as input to an 11-segment simulation model (Yeadon et al. 1990). For competitors 523 and 529, segmental inertia sets were available from Yeadon (1989b). For each of the remaining six competitors, the segmental inertia set was selected to be that of the four available which produced the smallest maximum deviation between the twist values obtained from simulation and from video from the start of the dive until the time the twist angle from video reached one revolution.

The relationship between the twist rate $p$, the tilt angle $\theta$ and the somersault rate $\Omega$ for an axially symmetric rigid body with principal moments of inertia $A, A$, and $C(A>C)$ is given in Yeadon (1993a) as:

$$
p=\Omega\left(\frac{A}{C}-1\right) \sin \theta
$$

Thus the angle of tilt between the longitudinal axis of the body and the invariable plane perpendicular to the angular momentum vector may be used to give the number of twists per somersault for a given body configuration providing that the two large principal moments of inertia are approximately equal. Since the angle of tilt is approximately proportional to the twist rate, it may therefore be used as a measure of the twisting potential present, independent of the actual configuration adopted. Twisting techniques may be defined as contact or aerial techniques depending on whether the twist is initiated during contact with the diving board or during the aerial phase (Yeadon, 1993b,c). The initial value of the tilt angle when the feet leave the board is a measure of the twist initiated during contact with the board, typically by turning the shoulders in the direction of twist before take-off. The subsequent increase in the tilt angle during the dive gives the contribution of aerial twisting techniques. Since the whole body principal moments of inertia are all distinct, nutation will occur so that the tilt angle oscillates as a function of the twist angle (Yeadon, 1986, 1993a). To minimize this effect, the tilt angle value was selected at the time that the one twist position was reached.

In order to partition the aerial contribution to the tilt angle into a symmetry contribution and contributions associated with asymmetries of arms, chest and hips, the configurational angles obtained from video were modified. These modifications comprised the systematic removal of asymmetries of the arms, chest and hips about the sagittal plane. Arm movement was made symmetrical by having the left arm mirror the recorded movement of the right arm and vice versa, chest torsion relative to the thorax was eliminated, and hip movement was restricted by only permitting flexion parallel to the sagittal plane. The systematic removal of arm, chest and hip asymmetries provided 12 configuration sequences ranging from the original movement to a sequence with complete left-right configurational symmetry. The tilt angle produced at the time corresponding to one twist in the original movement was determined from simulations based upon the modified configurations. Since arm asymmetries were removed in two ways, the tilt values were averaged in these simulations to give a total of eight tilt values for the modified simulations. The tilt contribution due to symmetry was calculated as the amount that the tilt angle in the simulation, in which left-right symmetry was maintained, rose above the initial value.

The tilt associated with asymmetrical arm movement could be calculated as the difference between the tilt values for the simulations of complete symmetry and of symmetrical chest and hips. It could also be calculated as the difference between the tilt values for the simulations corresponding to arm symmetry and the original configuration. The average of the tilt values obtained in these two ways was taken as the tilt contribution from asymmetrical arm movement. The other contributions to the tilt angle were calculated in a similar way as described in Yeadon (1993d). 


\section{Results}

Since the interpolated information sets from the two cameras were synchronized using the displacement data, the accuracy of the synchronization is dependent upon the accuracy of the displacement data. In order to determine the effect of the digitization error, the synchronization procedure was carried out using the two digitizations of each view. The differences in synchronization were used to give an estimate of the synchronization error at the start and end of each dive. These error estimates of the synchronization had a mean values of $0.0025 \mathrm{~s}$. The mean of the error estimates in the co-ordinate values obtained from the repeated digitization was $0.022 \mathrm{~m}$, which is comparable with the values obtained by Angulo and Dapena (1992) for video digitization with an $8 \mathrm{~m}$ field of view. The mean of the error estimates of the orientation and configuration angles was $2.0^{\circ}$.

The differences between the values of the orientation angles obtained from the simulation and video were evaluated at the time for which the video value of the twist angle was $1.0 \mathrm{rev}$. The mean errors were $0.02 \mathrm{rev}$ for somersault, $2.3^{\circ}$ for tilt and $0.04 \mathrm{rev}$ for twist. Since the inertia parameters for six of the divers were chosen from the four available sets on the basis of agreement between the twist values of simulation and video, the twist error value is likely to be an underestimate, although it could be argued that, had the individual's own inertia parameters been avaialble, the simulation error would have been smaller. When the twist errors were averaged for all four interia sets, a value of 0.08 rev was obtained. Figure 3 presents a comparision of video and simulation sequences for competitor 529, for whom anthropometric data were available.

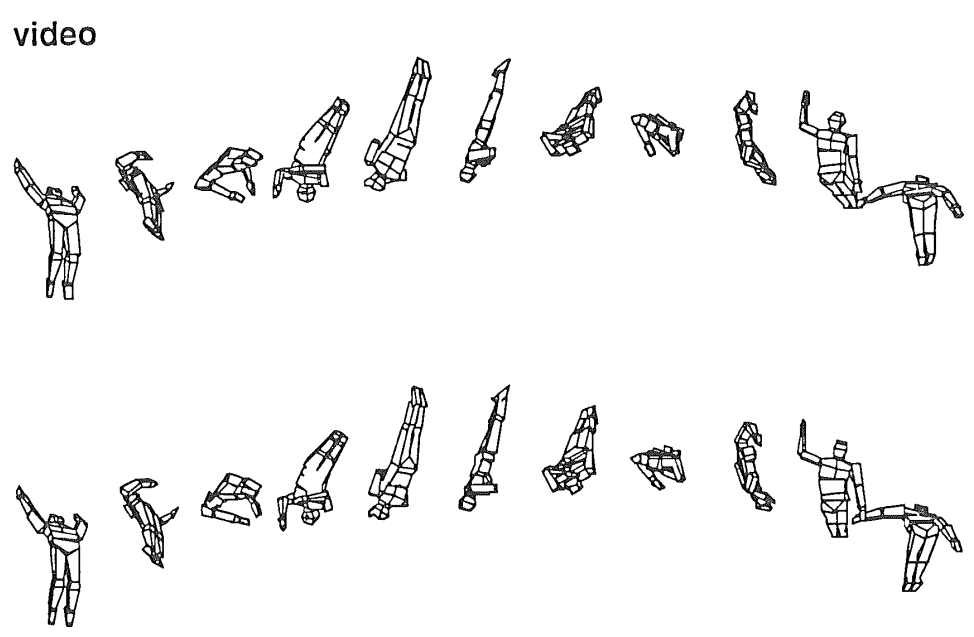

simulation

Figure 3: Comparison of video and simulation for competitor 529.

Tilt contributions were evaluated for the time at which the original simulation reached a twist value of $1 \mathrm{rev}$. This twist value was chosen to allow time for the tilt angle to approach its maximum value and to minimize the effect that nutation has on the tilt angle at times which do not correspond to integral multiples of half twists. Figure 4 shows tilt angle as a function of twist angle for competitor 661. It can be seen that the tilt angle rises rapidly during the first part of the twist and then remains approximately constant for the remainder of the twist. The variation in the tilt angle arising from nutation is evidently small, presumably since the configuration adopted had the two large principal moments of inertia approximately equal. This same pattern was evident in all the divers. The final portion of each dive, in which the tilt angle falls as preparation is made for entry, was not digitized, since the field of view only included the part of the dive above the diving board. Table 1 presents the contributions made to the tilt angle at the one twist position in each of the eight dives. The contact contribution is the initial tilt angle between the longitudinal axis and the invariable plane perpendicular to the angular momentum vector evaluated at the moment of take-off. The symmetry contribution indicates 
Table 1: Tilt contributions (in degrees) in reverse $1 \frac{1}{2}$ somersault dives with $2 \frac{1}{2}$ twists

\begin{tabular}{lcccccccc}
\hline Diver & Score & Contact & Symmetry & Arms & Chest & Hips & Total & Actual \\
\hline 529 & 62.10 & 3 & 1 & 4 & 1 & 5 & 14 & 14 \\
661 & 63.51 & 4 & 1 & 13 & 1 & 5 & 23 & 24 \\
523 & 59.40 & 4 & 3 & 9 & -1 & 6 & 22 & 22 \\
397 & 70.47 & 5 & 2 & 3 & 5 & 6 & 21 & 21 \\
219 & 60.30 & 7 & 2 & 13 & -1 & 2 & 23 & 24 \\
049 & 68.40 & 9 & 3 & 7 & 1 & 3 & 22 & 23 \\
528 & 56.55 & 10 & 1 & 6 & 0 & 4 & 20 & 20 \\
391 & 64.80 & 13 & 2 & 5 & -2 & 2 & 20 & 21 \\
$\bar{x}$ & 63.19 & 7 & 2 & 7 & 1 & 4 & 21 & 21 \\
\hline
\end{tabular}

Note: Contributions have been rounded to the nearest degree.

how much the tilt angle increases from its initial value during a simulation that enforces symmetry of the arms, chest and hips about the sagittal plane. The contributions listed under arms, chest and hips arise from asymmetrical arm movements, torsion of the chest and lateral flexion at the hips.

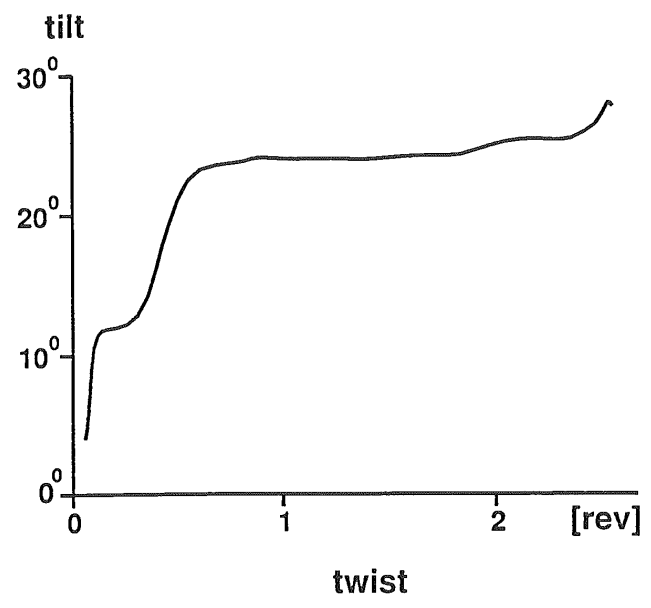

Figure 4: Tilt as a function of twist angle for competitor 661.

For each of the eight dives analysed, the sum of the tilt contributions (as listed under 'total') was within $1.4^{\circ}$ of the tilt value obtained from the unmodified simulation. It should be noted that the values in Table 1 have been rounded to the nearest degree so that the value under the 'total' heading is not always exactly the same as the sum of the stated individual values.

Competitor 391 was the only diver having a contact contribution which accounted for more than half of the total tilt. In the remaining seven cases, aerial techniques accounted for the majority of the contributions to the tilt angle. The contact contributions were all positive, however, ranging from $3^{\circ}$ to $13^{\circ}$ with a mean contact contribution of $6.9^{\circ}$. Symmetry contributions were all positive and ranged from $1^{\circ}$ to $3^{\circ}$. Asymmetrical arm movements made contributions ranging from $3^{\circ}$ to $13^{\circ}$ with a mean value of $7.4^{\circ}$. Chest contributions were small for all competitors except one with $5^{\circ}$. The negative chest contributions shown in Table 1 could possibly have arisen from an initial torsion of the chest in the direction of twist, during the take-off phase, which was reversed during flight causing the tilt angle to fall. In view of the small magnitude of the values, however, such contributions do not appear to be of much importance. Hip contributions ranged from $2^{\circ}$ to $6^{\circ}$ with a mean value of $4.1^{\circ}$.

Figure 5 shows a graphics sequence of competitor 391 who had a large contact contribution of $13^{\circ}$. The effect of the contact twist is evident in the first image of the sequence, which shows that the body had already twisted at take-off. Figure 5 also shows graphics sequences of competitors 661 and 397 who had large arm and hip contributions $13^{\circ}$ and $6^{\circ}$, respectively. 

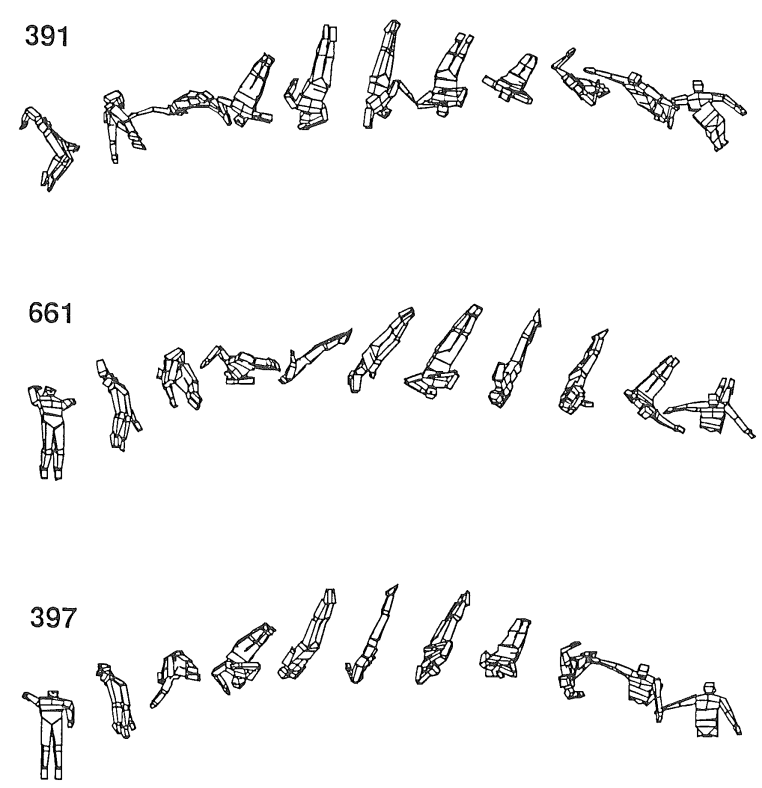

Figure 5: Graphics sequences of competitors 391, 661 and 397.

\section{Discussion}

The results may be interpreted as indicating that seven of the eight divers obtained the majority of their tilt from the use of aerial techniques. The major aerial techniques used were asymmetrical arm and hip movement. These findings are in agreement with those from an earlier study of elite divers (Yeadon, 1989b). One point of difference is that in the present study the mean contribution of contact techniques was considerably larger. A problem associated with large contact contributions is that there will be a tendency for the body to be tilted at entry. In Fig.5, it can be seen that competitor 391, who had a contact contribution of $13^{\circ}$, also had a large tilt angle at the completion of the twist, whereas competitors 661 and 397 were tilted to a much smaller degree.

It is clear that a number of divers at this level have substantial contributions from twist initiated during the contact phase. Whether their technique would improve if they reduced this contact contribution is not clear, since the extent to which twist can be initiated from the board without posing problems for the adjustment prior to entry has yet to be established. Some indication of this could be obtained by analysing the final phase of twisting dives in which the tilt angle is reduced to slow the twist rate and to give a more vertical entry. It is to be expected that contact contributions above a certain level will lead to less than ideal entry conditions, although the scores for each dive shown in Table 1 do not show any clear dependence upon the type of twisting technique used. However, since seven of the eight divers had a larger contribution from aerial rather than contact twisting techniques, there is evidence that elite divers use aerial techniques in preference to contact techniques.

\section{Acknowledgements}

This study was made possible by a grant from the National Coaching Foundation (UK) and by the cooperation of the organizers of Universiade ' 91.

\section{References}

Angulo, R.M. and Dapena, J (1992). Comparison of film and video techniques for estimating threedimensional co-ordinates with a large field. International Journal of Sport Biomechanics, 8, 145-151. 
Bartec, H. and Dowell, L. (1982). A cinematographical analysis of twisting about the longitudinal axis when performers are free of support. Journal of Human Movement Studies, 8, 41-54.

Lanouc, F. (1936). Mechanics of fancy diving. Unpublished master's thesis, Springfield College.

McCormick, G.P. (1954). A kinesiological study of four divers executing the full twisting forward one and one-half somersault. Unpublished master's thesis, University of Southern California.

McDonald, D. (1961). How does a man twist in the air? New Scientist, 10, 501-503.

Mood, D.P. (1968). A mechanical analysis of six twisting dives. Unpublished master's thesis, University of Iowa.

Pike, N.L. (1980). Computer simulation of a forward, full twisting dive in a layout position. Unpublished doctoral dissertation, Pennsylvania State University.

Sanders, R.H. and Wilson, B.D. (1986). Twisting techniques of elite springboard divers. Excel, 3, 9-11.

Van Gheluwe, B. (1981). A biomechanical simulation model for airborne twist in backward somersaults. Journal of Human Movement Studies, 7, 1-22.

Winter, F.W. (1966). A photographic analysis of selected twisting dives. Unpublished master's these, Springfield College.

Wood, G.A. and Jennings, L.S. (1979). On the use of spline functions for data smoothing. Journal of Biomechanics, 12, 477-479.

Yeadon, M.R. (1986). The biomechanics of twisting somersaults. In Proceedings of the North American Conference on Biomechanics pp. 33-34. Montreal: Canadian Society for Biomechanics.

Yeadon, M.R. (1989a). A method for obtaining three-dimensional data on ski jumping using pan and tilt cameras. International Journal of Sport Biomechanics

Yeadon, M.R. (1989b). Application of Computer Simulation for the Modification of Twisting Techniques of High Performance Divers. Report for Sport Canada Applied Research Progam. Gloucester: Sport Informance Resource Centre.

Yeadon, M.R. (1990a). The simulation of aerial movement - I: The determination of orientation angles from film data. Journal of Biomechanics, 23, 59-66.

Yeadon, M.R. (1990b). The simulation of aerial movement - II: A mathematical inertia model of the human body. Journal of Biomechanics, 23, 67-74.

Yeadon, M.R. (1990c). The simulation of aerial movement - III: The determination of the angular momentum of the human body. Journal of Biomechanics, 23, 75-83.

Yeadon, M.R. (1993a). The biomechanics of twisting somersaults. Part I: Rigid body motions. Journal of Sports Sciences, 11, 187-198.

Yeadon, M.R. (1993b). The biomechanics of twisting somersaults. Part II: Contact Twist. Journal of Sports Sciences, 11, 199-208.

Yeadon, M.R. (1993c). The biomechanics of twisting somersaults. Part III: Aerial twist. Journal of Sports Sciences, 11, 209-218. 
Yeadon, M.R. (1993d). The biomechanics of twisting somersaults. Part IV: Partitioning performance using the tilt angle. Journal of Sports Sciences, 11, 219-225.

Yeadon, M.R. and Dunn, E.G. (1987). A method for determining the contributions of aerial twisting techniques to performances of twisting somersaults. In Abstracts of the Eleventh Annual Meeting of the American Society of Biomechanics. Journal of Biomechanics, 20, 914.

Yeadon, M.R., Atha, J. and Hales, P.D. (1990). The simulation of aerial movement - IV: A computer simulation model. Journal of Biomechanics, 23, 85-89. 